\title{
Comportamento suicida na pandemia por COVID-19: Panorama geral
}

\author{
Suicide behavior in pandemia by COVID-19: General overview \\ Comportamiento suicida en la pandemia por COVID-19: Visión general
}

Recebido: 09/05/2021 | Revisado: 11/05/2021 | Aceito: 11/05/2021 | Publicado: 15/05/2021

\author{
Alice Barbosa Nascimento \\ ORCID: https://orcid.org/0000-0001-7373-5954 \\ Universidade Tiradentes, Brasil \\ E-mail: alicebnasc@hotmail.com \\ Juliana Leal Freitas Maia \\ ORCID: http://orcid.org/0000-0002-1751-4572 \\ Universidade Tiradentes, Brasil \\ E-mail: juliana.maia@ souunit.com.br
}

\begin{abstract}
Resumo
O propósito deste estudo foi realizar uma revisão bibliográfica com o objetivo de avaliar o comportamento suicida durante a pandemia por COVID-19. Sendo assim, foi realizada uma busca nos bancos de dados do PubMed e Scielo com os artigos científicos publicados entre janeiro de 2020 e abril de 2021. Os descritores utilizados foram Suicide e Coronavírus. Após aplicar os critérios de inclusão e exclusão, foram selecionados 37 artigos. A maioria dos estudos revisados demonstrou que a pandemia exacerbou alguns fatores de risco para o comportamento suicida, como a presença de transtorno psiquiátrico associado, isolamento social, desemprego, uso de álcool, violência doméstica, estigma social, notícias negativas, doenças neurológicas, transtornos do sono, acesso restrito a serviços de saúde e outros. Dessa forma, espera-se um aumento proporcional nas taxas de comportamento suicida durante e após o cenário atual da COVID-19. Ainda são necessárias mais pesquisas na área que contribuam para o reconhecimento precoce de fatores de risco que interferem diretamente no comportamento suicida. É importante que novos estudos foquem no desenvolvimento de estratégias de enfrentamento mais definidas diante dessa problemática, a fim de minimizar o impacto social, econômico e na saúde pública referente ao aumento do comportamento suicida.
\end{abstract}

Palavras-chave: Suicídio; Pandemia; Saúde mental; Covid-19.

\begin{abstract}
The purpose of this study was to conduct a literature review with the objective of assessing suicidal behavior during the COVID-19 pandemic. Thus, a search was conducted in the PubMed and Scielo databases for scientific articles published between January 2020 and April 2021. The descriptors used were Suicide and Coronavirus. After applying the inclusion and exclusion criteria, 37 articles were selected. Most of the reviewed studies showed that the pandemic exacerbated some risk factors for suicidal behavior, such as the presence of associated psychiatric disorder, social isolation, unemployment, alcohol use, domestic violence, social stigma, negative news, neurological diseases, sleep disorders, restricted access to health services, and others. Thus, a proportional increase in rates of suicidal behavior is expected during and after the current COVID-19 scenario. Further research is still needed in the area that contributes to the early recognition of risk factors that directly interfere with suicidal behavior. It is important that further studies focus on developing more defined coping strategies to minimize the social, economic, and public health impact of increased suicidal behavior.
\end{abstract}

Keywords: Suicide; Pandemic; Mental health; Covid-19.

\section{Resumen}

El propósito de este estudio fue realizar una revisión bibliográfica con el fin de evaluar la conducta suicida durante la pandemia de COVID-19. Por ello, se realizó una búsqueda en las bases de datos PubMed y Scielo con artículos científicos publicados entre enero de 2020 y abril de 2021. Los descriptores utilizados fueron Suicidio y Coronavirus. Después de aplicar los criterios de inclusión y exclusión, se seleccionaron 37 artículos. La mayoría de los estudios revisados demostraron que la pandemia exacerbó algunos factores de riesgo de comportamiento suicida, como la presencia de un trastorno psiquiátrico asociado, aislamiento social, desempleo, consumo de alcohol, violencia doméstica, estigma social, noticias negativas, enfermedades neurológicas, trastornos del sueño, acceso restringido a los servicios de salud y otros. Por lo tanto, se espera un aumento proporcional en las tasas de conducta suicida durante y después del escenario actual de COVID-19. Aún se necesita más investigación en el área que contribuya al reconocimiento temprano de los factores de riesgo que interfieren directamente con la conducta suicida. Es importante que los nuevos estudios se enfoquen en el desarrollo de estrategias de afrontamiento más definidas ante este problema, 
con el fin de minimizar el impacto social, económico y de salud pública relacionado con el aumento de la conducta suicida.

Palabras clave: Suicidio; Pandemia; Salud mental; Covid-19.

\section{Introdução}

A doença por coronavírus 2019 (COVID-19) é uma síndrome respiratória aguda grave causada pelo coronavírus (SARS-CoV-2). O primeiro caso foi detectado em Wuhan na China em dezembro de 2019, a infecção evoluiu para uma ameaça à saúde pública, originando um desafio para todas as nações (Banerjee et al., 2021). Em decorrência da pandemia, cerca de um terço da população global está sob alguma forma de quarentena ou bloqueio (McIntyre \& Lee, 2020). Embora essas medidas possam ser críticas para mitigar a propagação desta doença, as consequências no comportamento suicida serão observadas a curto e longo prazo (Zalsman et al., 2020).

Há registros na literatura descritos por Sher (2020a) os quais demonstram que as infecções por coronavírus estão associadas à neurotropismo e a neuroinvasão, sendo assim apresentam efeitos neurobiológicos. Dados biológicos recentes sugerem que o SARS-CoV-2 pode afetar o sistema nervoso central (SNC) através de vias biológicas relacionadas aos comportamentos suicidas: sistema de inflamação, sistema renina-angiotensina e receptores de nicotina (Conejero et al., 2021). Dessa forma, as infecções virais também podem estar relacionadas a sintomas psiquiátricos como resultado direto da infecção do vírus no cérebro, mas na maioria das vezes está associada à ativação de uma poderosa resposta imune-inflamatória (Brietzke et al., 2020).

Sher (2020b) afirma que a pandemia COVID - 19 pode levar a exacerbação ou desenvolvimento de uso de substâncias, ansiedade, depressão e outros transtornos psiquiátricos, impactando no aumento das taxas de suicídio durante e após a pandemia. Para Sher (2020c), o impacto psicológico da COVID-19 é tão preocupante quanto os efeitos sobre a saúde física. O medo da incerteza, do isolamento social, do desconhecido, e das declarações paradoxais da saúde pública levam a altas taxas de depressão e ansiedade entre o público em geral e especialmente entre grupos populacionais vulneráveis. Com a rápida disseminação da crise da COVID-19, casos de suicídio ocasionados pela pandemia foram relatados em diversos países do mundo (Que et al., 2020).

O suicídio consiste em uma das principais causas de mortalidade com custos sociais e emocionais devastadores, sendo descrito como uma causa de morte geralmente evitável e um grande problema de saúde pública global (Moutier, 2020). Segundo a OMS, a cada 40 segundos, uma pessoa comete suicídio no mundo. Além disso, de acordo com as projeções realizadas, para cada suicídio consumado existem 20 tentativas (Aquila et al., 2020).

De acordo com Caballero-Domínguez et al. (2020) o comportamento suicida engloba intenção, ideação, plano e suicídio consumado. Os dados disponíveis mostram que em situações de quarentena e isolamento físico, o risco de suicídio aumenta significativamente (Thakur \& Jain, 2020). Além disso, a perda da rotina ocasiona uma redução drástica no contato social e físico com outras pessoas, acarretando em um aumento da sensação de desamparo e angústia, resultando em sofrimento psíquico (Mari \& Oquendo, 2020).

Segundo Ventriglio et al. (2020), distanciamento social é um termo muito utilizado nesse contexto, mas o que se entende na realidade é o distanciamento físico, pois em momentos como este precisamos da proximidade emocional e social com outros indivíduos. De fato, as conexões sociais auxiliam as pessoas a lidar com o estresse, regular as emoções e continuar resilientes durante tempos desafiadores (Conejero et al., 2021). As medidas de saúde pública impostas devido a COVID-19 podem ocasionar o desenvolvimento de anomia, que tem repercussões na saúde mental, nas relações sociais, e consequências adversas típicas, como aumento dos casos de suicídio (Bastiampillai et al., 2020).

As principais teorias do suicídio evidenciam o papel importante que as conexões sociais exercem na prevenção do suicídio. De acordo com a teoria interpessoal de Joiner, o risco de suicídio torna-se maior quando o indivíduo experimenta uma 
pertença frustrada, que ocorre diante dos casos de isolamento social e uma sobrecarga percebida, quando percebe-se como um fardo para os outros (Levi-Belz \& Aisenberg, 2020). Ainda abordando esta temática, Durkheim propôs por meio de sua teoria que períodos de mudanças sociais, políticas ou econômicas, podem resultar em "suicídio anômico" proveniente da falta de conexão social e um aumento da preocupação com o próprio interesse (Bastiampillai et al., 2020).

Embora a COVID-19 sozinha possa não ser o único fator para o suicídio, o isolamento físico associado com a desconexão social e o sofrimento psíquico podem ser uma junção nociva de fatores de risco (Ammerman et al., 2020). Considerando que a pandemia causa sofrimento, suscetibilidade ao suicídio e aos transtornos psiquiátricos, as consequências para a saúde mental tendem a persistir por mais tempo e alcançar o pico depois da pandemia real (Levi-Belz \& Aisenberg, 2020). Com isso, a prevenção é o elemento fundamental na preservação da saúde mental dos indivíduos. Uma intervenção psicológica eficaz e adequada é primordial para proteger os pacientes do suicídio (Que et al., 2020).

\section{Metodologia}

Trata-se de um estudo que tem como base uma análise, por revisão bibliográfica, de artigos científicos disponíveis nas bases de dados Scielo e Pubmed publicados no período entre janeiro de 2020 a abril de 2021. O estudo tem como objetivo avaliar se houve aumento do comportamento suicida durante a pandemia por coronavírus. Os descritores utilizados foram Suicide e Coronavírus, consultados através dos Descritores em Ciências da Saúde (DeSCS). A busca dos materiais que abordaram a temática em questão resultou em um total de 367 estudos potencialmente elegíveis, sendo 360 artigos disponíveis no Pubmed e 7 artigos encontrados no Scielo. Um registro adicional foi obtido por meio da busca manual das listas de referência dos artigos incluídos e das publicações referentes ao Suicídio. Após a aplicação dos critérios de inclusão e exclusão foi selecionado um total de 37 artigos.

A presente revisão sistemática possui uma abordagem qualitativa de acordo com Gomes e Caminha (2013), por se tratar de uma síntese de estudos relacionados ao tema principal, com interpretação e agrupamento dos resultados de outros autores, mas sem a aplicação de análises estáticas de forma integral. Os dados qualitativos foram analisados por meio da técnica de análise do conteúdo em três etapas, a pré- análise; exploração do material e tratamento dos resultados obtidos e interpretação (Bardin, 2011).

Os critérios utilizados para inclusão dos artigos nesta revisão foram estudos publicados no último ano, que abordassem a temática compreendida, sendo esta a relação entre Suicídio e a pandemia de COVID-19. Outros critérios foram populações de todas as faixas etárias, sem restrição de localidade e publicados nos idiomas inglês, português e espanhol. Foram excluídos artigos duplicados e/ou não relacionados a temática proposta e aqueles que não apresentavam dados sobre o comportamento suicida na pandemia COVID-19. Os critérios de exclusão adicionais incluíram estudos de caso, comentários e artigos de opinião, que não atendessem aos critérios de inclusão da pesquisa.

\section{Resultados}

Para a análise dos dados, foi elaborado um quadro com a síntese das informações extraídas dos artigos selecionados, incluindo a autoria e ano de publicação, o objetivo do artigo e os principais resultados da revisão integrativa. 
Quadro 1: Estudos sobre o comportamento suicida durante a pandemia por COVID-19, objetivo do estudo e resultados.

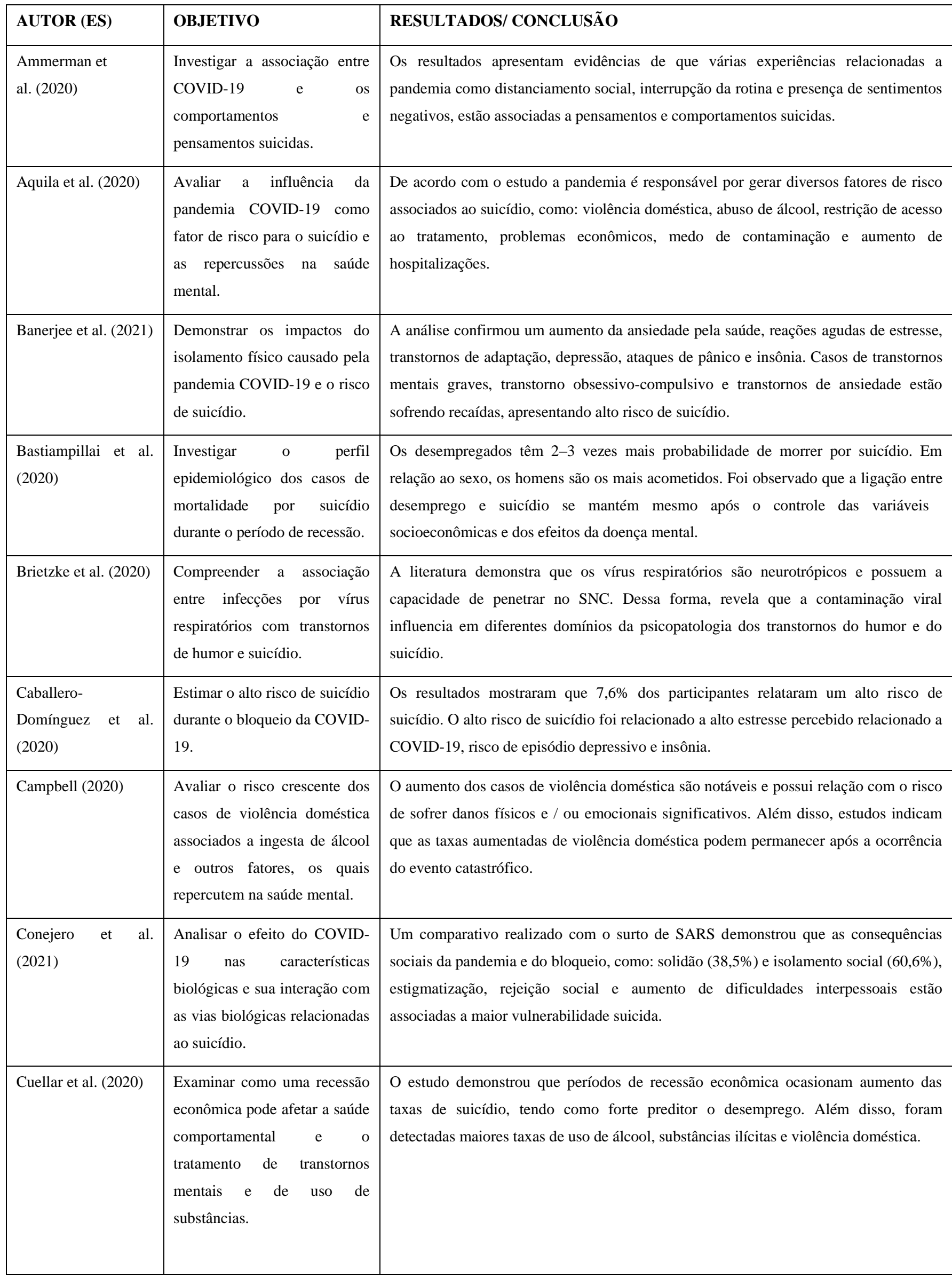




\begin{tabular}{|c|c|c|}
\hline Czeisler et al. (2020) & $\begin{array}{l}\text { Investigar a saúde mental, uso } \\
\text { de substâncias e ideação } \\
\text { suicida durante a pandemia. }\end{array}$ & $\begin{array}{l}\text { Na análise } 40,9 \% \text { dos entrevistados relataram pelo menos uma doença mental adversa } \\
\text { ou condição de saúde comportamental. Além disso, } 13,3 \% \text { relataram o início do uso de } \\
\text { substâncias. O aumento desses fatores causa elevação nas taxas de suicídio. }\end{array}$ \\
\hline Deady et al. (2020) & $\begin{array}{l}\text { Determinar como o } \\
\text { desemprego e as repercussões } \\
\text { financeiras podem interferir } \\
\text { nas taxas de suicídio. }\end{array}$ & $\begin{array}{l}\text { Após analisar dados relacionados com outras crises de saúde foi possível observar que } \\
\text { um aumento das taxas de desemprego coincidiu com um aumento nas taxas de suicídio } \\
\text { em ambos os sexos. De acordo com as projeções, na crise atual pode dobrar a taxa de } \\
\text { desemprego, causando conclusões preocupantes. }\end{array}$ \\
\hline $\begin{array}{l}\text { Fitzpatrick et al. } \\
(2020)\end{array}$ & $\begin{array}{l}\text { Observar a relação entre } \\
\text { vulnerabilidade social, risco } \\
\text { individual e recursos sociais/ } \\
\text { psicológicos com o suicídio. }\end{array}$ & $\begin{array}{l}\text { Quase } 15 \% \text { da amostra foram categorizados como alto risco de suicídio. As taxas não } \\
\text { são distribuídas uniformemente entre os subgrupos da população socialmente } \\
\text { vulneráveis, e existem diferentes fatores de risco que exacerbam ou mitigam o risco de } \\
\text { suicídio durante as crises de saúde pública. }\end{array}$ \\
\hline $\begin{array}{l}\text { Fountoulakis et al. } \\
(2021)\end{array}$ & $\begin{array}{l}\text { Investigar as mudanças na } \\
\text { ansiedade, angústia, ideação } \\
\text { suicida e suas relações com } \\
\text { uma série de variáveis sociais. }\end{array}$ & $\begin{array}{l}\text { Durante o estudo foi observado que a depressão clínica esteve presente em } 9,31 \% \text { da } \\
\text { amostra, enquanto } 8,5 \% \text { apresentavam sofrimento grave; o aumento da ansiedade } \\
\text { estava presente em mais de } 45 \% \text {. Os pensamentos suicidas aumentaram em } 10,40 \% \text {. }\end{array}$ \\
\hline Garcia et al. (2020) & $\begin{array}{l}\text { Avaliar o consumo excessivo } \\
\text { de álcool diante das mudanças } \\
\text { impostas pela pandemia } \\
\text { COVID-19. }\end{array}$ & $\begin{array}{l}\text { Em uma pesquisa aplicada no Brasil cerca de } 18 \% \text { da população relatou aumento do } \\
\text { uso de bebidas alcoólicas durante a pandemia. Além do risco para o suicídio, isso } \\
\text { dificulta a adesão das medidas preventivas, pois ocorre a redução da percepção de } \\
\text { risco, levando a uma má adesão às medidas de higiene e o uso incorreto das máscaras. }\end{array}$ \\
\hline Gunnell et al. (2020) & $\begin{array}{l}\text { Reconhecer os fatores de risco } \\
\text { mais prevalentes associados ao } \\
\text { suicídio e a importância das } \\
\text { medidas que mitiguem os } \\
\text { casos. }\end{array}$ & $\begin{array}{l}\text { Os resultados mostram que aqueles com transtornos psiquiátricos podem ter piora dos } \\
\text { sintomas e relatar novos agravos, especialmente ansiedade, depressão e estresse pós- } \\
\text { traumático. O estudo também demonstra que o aprisionamento, isolamento social e a } \\
\text { solidão contribuem para o risco de suicídio. }\end{array}$ \\
\hline Han et al. (2020) & $\begin{array}{l}\text { Investigar as sequelas de saúde } \\
\text { mental das pandemias } \\
\text { anteriores buscando } \\
\text { embasamento para prevenir as } \\
\text { tendências atuais. }\end{array}$ & $\begin{array}{l}\text { Os dados disponíveis sugerem que as sequelas de saúde mental do COVID-19 serão } \\
\text { semelhantes às de pandemias anteriores. Observando o grande predomínio de efeitos } \\
\text { negativos do isolamento, sendo os mais prevalentes angústia e ansiedade, bem como os } \\
\text { sintomas de estresse pós-traumático. }\end{array}$ \\
\hline $\begin{array}{l}\text { Hernández-Calle et } \\
\text { al. (2020) }\end{array}$ & $\begin{array}{l}\text { Avaliar o impacto do surto de } \\
\text { COVID-19 nas visitas } \\
\text { semanais ao departamento de } \\
\text { emergência } \\
\text { devido o } \\
\text { suicidar }\end{array}$ & $\begin{array}{l}\text { Com base na análise dos atendimentos psiquiátricos foi observado uma redução das } \\
\text { visitas de emergência relacionadas ao suicídio. Tornando evidente à evitação dos } \\
\text { pacientes do pronto-socorro durante crises suicidas, provavelmente por medo do } \\
\text { contágio ou restrições de acesso. }\end{array}$ \\
\hline Jefsen et al. (2020) & $\begin{array}{l}\text { Entender a associação entre } \\
\text { autoagressão e suicídio nos } \\
\text { indivíduos com transtorno } \\
\text { mental. }\end{array}$ & $\begin{array}{l}\text { Ficou evidente um aumento do suicídio/ automutilação em indivíduos com transtornos } \\
\text { mentais. Estão inseridos no alto risco portadores de transtornos psicóticos, transtornos } \\
\text { do humor, transtornos relacionados ao estresse e transtornos de personalidade. }\end{array}$ \\
\hline $\begin{array}{l}\text { Kawohl e Nordt } \\
(2020)\end{array}$ & $\begin{array}{l}\text { Entender a relação entre os } \\
\text { casos de COVID } 19, \\
\text { desemprego e as taxas de } \\
\text { suicídio. }\end{array}$ & $\begin{array}{l}\text { Com base nas projeções anteriores, foi observado que um aumento dos suicídios } \\
\text { precedeu o aumento real da taxa de desemprego. Além disso, espera-se um aumento da } \\
\text { busca de atendimento nos serviços de saúde mental. }\end{array}$ \\
\hline
\end{tabular}




\begin{tabular}{|c|c|c|}
\hline Leaune et al. (2020) & $\begin{array}{l}\text { Comparar o comportamento e } \\
\text { ideação suicidas antes da } \\
\text { pandemia de COVID-19 e } \\
\text { relacionar com o cenário atual. }\end{array}$ & $\begin{array}{l}\text { Os estudos observam um aumento da mortalidade por suicídio, principalmente em } \\
\text { adultos mais velhos. Já em relação aos atendimentos no departamento de emergência, } \\
\text { foi observado uma redução dos casos de tentativa de suicídio. Porém, os } \\
\text { comportamentos suicidas precisam ser observados anos depois dos surtos virais. }\end{array}$ \\
\hline Lennon (2020) & $\begin{array}{l}\text { Avaliar as projeções futuras } \\
\text { em relação aos casos de } \\
\text { ansiedade, depressão e } \\
\text { comportamentos suicidas. }\end{array}$ & $\begin{array}{l}\text { Com base nos dados de eventos prévios, as projeções futuras indicam para o cenário } \\
\text { atual um aumento esperado de } 27,4 \% \text { nas taxas de suicídio dos últimos anos desde } \\
\text { 2012. Além disso, nota-se um aumento na ansiedade e distúrbios do humor. }\end{array}$ \\
\hline $\begin{array}{l}\text { Levi-Belz } \\
\text { Aisenberg (2020) }\end{array}$ & $\begin{array}{l}\text { Demonstrar } \\
\text { relacionados com o risco de } \\
\text { suicídio em adultos mais } \\
\text { velhos durante e após a } \\
\text { COVID-19. }\end{array}$ & $\begin{array}{l}\text { No âmbito da COVID-19 foi possível observar que a desconexão social juntamente } \\
\text { com a depressão, solidão, pertença frustrada e peso percebido são fatores relacionados } \\
\text { com o risco aumentado de suicídio. }\end{array}$ \\
\hline $\begin{array}{l}\text { Mari e Oquendo } \\
(2020)\end{array}$ & $\begin{array}{l}\text { Analisar as consequências da } \\
\text { pandemia e os danos causados } \\
\text { na saúde mental dos } \\
\text { indivíduos. }\end{array}$ & $\begin{array}{l}\text { A pandemia pode causar sequelas na saúde mental de diversas formas, sendo } \\
\text { observado um primeiro impacto com a chegada repentina do vírus e o medo de } \\
\text { contágio. Além disso, outros fatores como a quarentena e a perda das conexões sociais, } \\
\text { altos índices de mortalidade e a crise econômica podem influenciar na saúde mental. }\end{array}$ \\
\hline $\begin{array}{l}\text { McIntyre e Lee } \\
(2020)\end{array}$ & $\begin{array}{l}\text { Observar o aumento dos casos } \\
\text { de suicídio decorrentes da } \\
\text { COVID-19. }\end{array}$ & $\begin{array}{l}\text { Obteve correlações significativas entre um aumento extremo nas taxas de desemprego } \\
\text { e as taxas de suicídio, projetando um aumento para } 14 \% \text { em } 2020 \text { e 13,6 \% em } 2021 . \\
\text { Revelando a urgência de priorizar o acesso à saúde mental. }\end{array}$ \\
\hline Moutier (2020) & $\begin{array}{l}\text { Identificar as ameaças da } \\
\text { COVID-19 à saúde mental da } \\
\text { população e o risco de } \\
\text { suicídio. }\end{array}$ & $\begin{array}{l}\text { Os entrevistados relataram efeitos prejudiciais em sua saúde mental com aumento dos } \\
\text { casos de } 39 \% \text { em maio para } 53 \% \text { em julho de } 2020 \text {. Além disto, estudos usando } \\
\text { métodos de autópsia psicológica descobriram que, entre os suicidas, } 85 \% \text { a } 95 \% \\
\text { provavelmente experimentaram doenças psiquiátricas que contribuíram para a morte. } \\
\text { Outros fatores também apresentam risco, sendo eles o aumento nas vendas de álcool e } \\
\text { as mensagens divulgadas na mídia. }\end{array}$ \\
\hline Que et al. (2020) & $\begin{array}{l}\text { Investigar os fatores de risco } \\
\text { do suicídio durante a } \\
\text { pandemia. }\end{array}$ & $\begin{array}{l}\text { A análise observou que fatores como a recessão econômica, desemprego, desconexão } \\
\text { social, transtornos mentais, notícias negativas e tentativas de suicídio; necessitam de } \\
\text { intervenção oportuna e precoce, pois serão eficazes para reduzir o número de suicídios. }\end{array}$ \\
\hline Rana (2020) & $\begin{array}{l}\text { Avaliar o aumento das taxas } \\
\text { de suicídio entre os idosos no } \\
\text { período da pandemia. }\end{array}$ & $\begin{array}{l}\text { Notou-se um aumento nas taxas de suicídio nos idosos que vivem sozinhos e } \\
\text { encontram-se desprotegidos devido à falta de apoio social no cenário atual. Cerca de } \\
10 \% \text { a } 20 \% \text { deles estão sofrendo de solidão e desolação mental. }\end{array}$ \\
\hline Sheffler et al. (2021) & $\begin{array}{l}\text { Observar os impactos } \\
\text { interpessoais e psicológicos da } \\
\text { COVID-19 no risco de } \\
\text { suicídio na terceira idade. }\end{array}$ & $\begin{array}{l}\text { Devido a COVID-19 algumas condições como o estresse, acesso restrito a cuidados de } \\
\text { saúde e distanciamento social, tendem a exacerbar os riscos já existentes de suicídio } \\
\text { nos idosos. }\end{array}$ \\
\hline Sher (2020a) & $\begin{array}{l}\text { Identificar o risco de suicídio } \\
\text { nos indivíduos sobreviventes } \\
\text { da COVID-19. }\end{array}$ & $\begin{array}{l}\text { Uma revisão recente mostrou que alterações no SNC como manifestações neurológicas } \\
\text { estão presentes em cerca de } 25 \% \text { dos sobreviventes e possuem associação com um } \\
\text { risco aumentado de suicídio. Ainda no estudo, os transtornos relacionados ao estresse, } \\
\text { depressão, transtornos do sono e outros estão associados ao comportamento suicida. }\end{array}$ \\
\hline Sher (2020b) & $\begin{array}{l}\text { Avaliar os fatores de risco que } \\
\text { influenciam no suicídio, } \\
\text { visando a redução dos casos }\end{array}$ & $\begin{array}{l}\text { Por meio do estudo foi observado que os transtornos mais associados foram depressão, } \\
\text { ansiedade e estresse. Os dados demonstram que o aparecimento ou agravamento de } \\
\text { transtornos psiquiátricos é um fator de risco que pode levar a um aumento de suicídios. }\end{array}$ \\
\hline
\end{tabular}


Research, Society and Development, v. 10, n. 5, e59410515923, 2021

(CC BY 4.0) | ISSN 2525-3409 | DOI: http://dx.doi.org/10.33448/rsd-v10i5.15923

\begin{tabular}{|c|c|c|}
\hline & durante e após a pandemia. & \\
\hline Sher (2020c) & $\begin{array}{l}\text { Discutir o impacto da } \\
\text { pandemia COVID-19 na saúde } \\
\text { mental da população em geral. }\end{array}$ & $\begin{array}{l}\text { Aproximadamente mais de } 25 \% \text { dos participantes do estudo relataram sintomas de } \\
\text { ansiedade moderados a graves. Os sintomas depressivos, em média, eram elevados. } \\
\text { Este estudo também mostrou que o medo da COVID-19 parecia estar centralizado nas } \\
\text { regiões com o maior número de casos. }\end{array}$ \\
\hline $\begin{array}{l}\text { Stockwell et al. } \\
(2021)\end{array}$ & $\begin{array}{l}\text { O impacto do álcool e sua } \\
\text { influência na saúde mental } \\
\text { durante a pandemia COVID- } \\
19 .\end{array}$ & $\begin{array}{l}\text { O estudo demonstra que o álcool é um fator de risco significativo para suicídio. Além } \\
\text { disso, o aumento da ingesta durante o período atual pode causar persistência desse } \\
\text { hábito no futuro. Ainda nesse contexto, o álcool dificulta o cumprimento das } \\
\text { precauções básicas para controle da pandemia. }\end{array}$ \\
\hline Thakur e Jain (2020) & $\begin{array}{l}\text { Avaliar os possíveis fatores e } \\
\text { preditores associados ao } \\
\text { suicídio na pandemia. }\end{array}$ & $\begin{array}{l}\text { Com base nos dados relatos sobre suicídio, podemos antecipar o efeito propagador } \\
\text { desse vírus em eventos suicidas em todo o mundo. Alguns fatores preditores estão } \\
\text { envolvidos, são eles: isolamento social, estresse, recessão econômica e discriminação. }\end{array}$ \\
\hline $\begin{array}{l}\text { Ventriglio et al. } \\
(2020)\end{array}$ & $\begin{array}{l}\text { Analisar os estágios de } \\
\text { manifestações psicológicas } \\
\text { diante da pandemia por } \\
\text { COVID-19. }\end{array}$ & $\begin{array}{l}\text { É muito provável que os diversos estágios da pandemia produzam um aumento não } \\
\text { apenas nas condições psiquiátricas, como depressão, pânico e ansiedade, mas também } \\
\text { nas taxas de suicídio. }\end{array}$ \\
\hline Wand et al. (2020) & $\begin{array}{lr}\text { Observar as ligações entre } \\
\text { suicídio em pessoas idosas e a } \\
\text { pandemia } & \text { COVID-19, } \\
\text { considerando } & \text { diferentes } \\
\text { desafios e } & \text { contextos } \\
\text { socioculturais. } & \end{array}$ & $\begin{array}{l}\text { Uma análise com idosos chineses revelou que } 39,2 \% \text { estavam com baixo-astral e } 4,4 \% \\
\text { relataram a ideação suicida, indicando que o cenário atual implica em um aumento de } \\
\text { fatores que predispõem ao suicídio. Necessitando de serviço especializado de } \\
\text { atendimento. }\end{array}$ \\
\hline $\begin{array}{l}\text { Wasserman et al. } \\
(2020)\end{array}$ & $\begin{array}{l}\text { Demonstrar como o } \\
\text { distanciamento físico pode } \\
\text { afetar pessoas com problemas } \\
\text { de saúde mental. }\end{array}$ & $\begin{array}{l}\text { O estudo demonstra que o distanciamento físico é prejudicial, pois evoca sentimentos } \\
\text { negativos de exclusão, medo e solidão. Esses sentimentos são mais prevalentes em } \\
\text { grupos vulneráveis, muitos deles também com alto risco de comportamentos suicidas. }\end{array}$ \\
\hline Zalsman et al. (2020) & $\begin{array}{l}\text { Observar os principais fatores } \\
\text { associados ao suicídio durante } \\
\text { a pandemia. }\end{array}$ & $\begin{array}{l}\text { Demonstra que a maior vulnerabilidade diante dos casos de desemprego, mortes, } \\
\text { incertezas e outros fatores, são capazes de resultar em uma confluência de fatores de } \\
\text { risco que impactam no aumento das taxas de suicídio e tentativa de suicídio a longo } \\
\text { prazo. }\end{array}$ \\
\hline
\end{tabular}

Fonte: Autores.

Em relação aos resultados, os estudos presentes no Quadro 1 demonstram que no cenário pandêmico atual é possível observar um aumento dos fatores que influenciam no risco de comportamento suicida, sendo eles o desemprego, transtorno psiquiátrico associado, estigma social, notícias negativas, isolamento social , uso de álcool, transtornos do sono, violência doméstica, acesso restrito a serviços de saúde, doenças neurológicas e outros, ratificando assim a importância da análise destas repercussões em todas as faixas etárias durante e após a pandemia COVID-19.

\section{Discussão}

Com o desdobramento da pandemia COVID - 19, os pesquisadores começaram a notar um alto potencial de suscetibilidade a consequências extremas para a saúde mental, incluindo ideação e tentativas de suicídio (Fitzpatrick et al., 2020). A pandemia é um tipo de catástrofe biológica, sendo um acontecimento extremamente traumático que pode aumentar o 
risco de suicídio (Que et al., 2020). Para Caballero-Domínguez et al. (2020), a taxa de suicídio é considerada um indicador proxy global de transtornos mentais nos indivíduos em geral.

De acordo com a literatura, Sher (2020b) afirma que os sobreviventes da COVID-19 continuamente apresentam transtorno de estresse pós-traumático, insônia, depressão, ansiedade e sintomas obsessivo-compulsivos. Esses dados revelam risco de suicídio, pois a maioria dos suicidas tem uma doença psiquiátrica diagnosticável (Sher, 2020c). Para Que et al. (2020), a associação entre suicídio e transtornos psiquiátricos está bem estabelecida.

Como descrito por Sher (2020a), transtornos neurológicos, psiquiátricos, e outros estão associados a risco elevado de suicídio. Em consonância, Banerjee et al. (2021) revelam que a COVID-19 levou ao aumento do risco de transtornos psiquiátricos, trauma crônico e estresse, que eventualmente aumentam o comportamento suicida e a tendência suicida. Sendo assim, a crise do COVID-19 é, certamente, um 'duplo golpe' acarretando no aumento da suscetibilidade.

Para Wasserman et al. (2020), o processo suicida pode ser impulsionado devido a uma interação entre os sentimentos caóticos dos indivíduos e a notícia de que "o distanciamento social é essencial” circulando em todos os âmbitos da sociedade. Em consonância, Gunnell et al. (2020) afirmam que reportagens irresponsáveis da mídia podem levar a picos de suicídios, pois a exposição repetida de relatos sobre a crise pode aumentar o risco de suicídio. Como descrito por Moutier (2020), é importante que durante a COVID-19 a mídia evite determinadas consequências não intencionais relacionadas ao suicídio, mantendo as mensagens focadas no suicídio como uma causa de morte evitável e oferecendo recursos de apoio e ajuda.

Segundo Conejero et al. (2021), as conexões sociais são fundamentais na eficácia coletiva e promovem a esperança, como também as oportunidades de compartilhar altruísmo, emoções positivas, compaixão e gratidão. De fato, todas as formas de apoio psicológico proporcionam a resiliência psicológica e reforça a psico-neuroimunidade contra COVID-19. Com base nisso, Lennon (2020) evidencia que o isolamento social e o status socioeconômico são grandes preditores de suicídio e seguramente podem resultar em picos na pandemia atual.

Como descrito por Gunnell et al. (2020), nesse período de isolamento domiciliar o acesso aos meios é um importante fator de risco para o suicídio, pois no ambiente atual, os meios letais como pesticidas, analgésicos e armas de fogo podem estar mais disponíveis no domicílio. Para Ammerman et al. (2020), esse maior acesso é um preditor vinculado as mortes por suicídio. Os dados disponibilizados por Moutier (2020) demonstram que nos EUA, as compras de armas de fogo aumentaram 85\% no início da COVID-19, em comparação com os anos anteriores no mesmo período.

De acordo com Czeisler et al. (2020) um estudo realizado nos EUA revelou que durante a pesquisa alguns indivíduos questionados relataram o aumento ou início do uso de substâncias para enfrentar as emoções ou estresse relacionados a pandemia. Para Stockwell et al. (2021) uma substância que demonstrou níveis elevados durante o período de isolamento social forçado foi o álcool, sendo este um fator de risco significativo para depressão e suicídio. Além disso, Campbell (2020) afirma que o abuso de álcool, é um fator de risco bastante relatado para violência familiar e tem sido associado à falta de apoio social e a um acúmulo de eventos estressantes.

Na visão de Garcia et al. (2020) o uso de álcool em situações de estresse ocorre por seu efeito depressor do SNC, que, em uma primeira fase, parece causar relaxamento. Além disso, há evidências de que o risco de dependência de álcool aumenta à medida que as pessoas são expostas a catástrofes naturais. Como exposto por Gunnell et al. (2020), a pandemia pode afetar adversamente outros fatores desencadeantes do suicídio, pois durante o período do confinamento o consumo de álcool e a violência doméstica podem aumentar. No seu estudo Campbell (2020) alega que o Brasil estima que os relatórios de violência doméstica aumentaram 40-50\% durante o período da pandemia da COVID-19.

Segundo Thakur e Jain (2020), a quarentena e o isolamento afetam a vida social e desenvolve medo psicológico e sensação de estar preso por um período de tempo incerto. Com base nisso, Sheffler et al. (2021) demonstram que o potencial estresse prolongado devido à pandemia de COVID-19 pode influenciar no funcionamento imunológico e neurológico - 
agravando o risco de suicídio. Nesse contexto Fountoulakis et al. (2021) ratificam que as mudanças nos hábitos e rotinas diárias, nas condições de trabalho, assim como no comportamento social, devem causar mais estresse, especialmente com a chance de uma iminente crise econômica e provável desemprego.

Para Deady et al. (2020), o desemprego está associado a um aumento de duas a três vezes no risco relativo de morte por suicídio em comparação com indivíduos empregados, também foi visto que picos no desemprego estão associados a aumentos compatíveis nas taxas de suicídio da população. De acordo com Cuellar et al. (2020), a maioria dos indicadores de saúde melhoram com o aumento do desemprego, incluindo uma redução nos acidentes e mortes de trânsito e um aumento no exercício físico, porém o suicídio é uma ressalva importante.

Conforme descrito por McIntyre e Lee (2020), as taxas de suicídio são altamente vulneráveis aos indicadores macroeconômicos, em especial o desemprego. Um estudo publicado recentemente por Sher (2020c) mostra que as perdas de empregos relacionadas à crise da COVID-19 resultarão entre 2.135 e 9.570 suicídios por ano em todo o mundo. O trabalho realizado por Cuellar et al. (2020) sugere que cada aumento de ponto percentual no desemprego está relacionado a um aumento de $0,79 \%$ nos casos de suicídios entre indivíduos com idade inferior a 65 anos.

De acordo com Bastiampillai et al. (2020), as taxas de suicídio durante a pandemia COVID-19 podem aumentar em 15\%, com homens em idade produtiva, sendo o grupo de maior risco. Ainda nesse contexto, Jefsen et al. (2020) demonstram que um estudo realizado com 102 pacientes adultos de serviços psiquiátricos da região central da Dinamarca, evidenciou que 74 pacientes exibindo lesões autoprovocadas relacionadas à pandemia tinha uma idade mediana de 29,8 anos e $77 \%$ eram mulheres.

No contexto atual da pandemia os principais temas associados como supostas razões para o suicídio incluíram o medo de contrair a infecção, o medo da transmissão e o medo da quarentena (Mari \& Oquendo, 2020). Com base nisso, Rana (2020) demonstrou que na Índia mais de 300 suicídios em idosos foram relatados durante o bloqueio associadas ao tormento mental. Com base nos dados, 80 pessoas se mataram por medo de serem infectadas e pela solidão. De acordo com Ammerman et al. (2020), uma pesquisa recente nos EUA com 907 participantes, observou que $45 \%$ dos indivíduos relatavam ideação suicida e ligaram seus pensamentos a pandemia. Além disso, o mesmo estudo descobriu que a exposição intencional ao COVID-19 está sendo usada como um método de suicídio.

Conforme a análise de Han et al. (2020), tendo como base os relatos de surtos respiratórios anteriores, é possível presumir que o surto atual de COVID-19 pode causar um aumento importante e global de problemas de saúde mental. Para Lennon (2020), parte do impacto residual pode contribuir para outra pandemia contínua de uma variante diferente - o suicídio. De acordo com Leaune et al. (2020), através de uma análise em pacientes dos Centros de Tratamento de Ebola foi observada a persistência da ocorrência de tentativas de suicídio cinco, onze e doze meses após a alta do quadro.

Com base nisso, Gunnell et al. (2020) relatam que indivíduos em crises suicidas necessitam de um cuidado minucioso. No cenário atual existem algumas barreiras para o atendimento psiquiátrico, pois alguns pacientes apresentam receio que os serviços estejam sobrecarregados e ou acreditam que comparecer às consultas presenciais possa colocá-los em risco de contágio. Para Sher (2020c), é fundamental que sejam implementadas medidas proativas para reduzir o impacto prejudicial da pandemia da COVID-19 na saúde mental.

Ademais, na visão de Hernández-Calle et al. (2020) os serviços de psiquiatria devem ressaltar fortemente a vigilância do acesso dos pacientes ao atendimento de emergência e a implantação de estratégias alternativas de extensão. Conforme Deady et al. (2020), o engajamento de novas tecnologias na luta contra o suicídio pode representar uma nova ferramenta valiosa. Wand et al. (2020) afirmam que, embora a quarentena seja essencial para reduzir a disseminação da pandemia, podem ser tomadas medidas para minimizar os fatores associados à saúde mental fragilizada. 
Os efeitos psiquiátricos da crise COVID-19 se tornarão gradativamente evidentes nos próximos meses e anos à medida que se manifestam as consequências da ansiedade crônica, sofrimento prolongado, distanciamento físico, solidão, morte de amigos e familiares e perdas de emprego (Sher, 2020c). Nesse contexto, Zalsman et al. (2020) demonstram que as consequências da COVID-19 podem levar ao surgimento ou ter efeitos exacerbantes em pensamentos e comportamentos suicidas. Portanto, esperamos um fardo adicional para nosso sistema de saúde mental, e a comunidade médica deve se preparar para esse novo desafio. (Kawohl \& Nordt, 2020).

\section{Conclusão}

O presente estudo se propôs a avaliar o comportamento suicida diante dos impactos causados pela pandemia da COVID-19 na saúde mental da população. Compreendeu-se que, as medidas de contenção da doença trouxeram algumas repercussões negativas, principalmente em ambientes psicossociais mais vulneráveis, diante das situações que precisam de quarentena e isolamento social.

O comportamento suicida possui uma abordagem multifatorial, já que diversos fatores estão envolvidos no desenvolvimento ou exacerbação dos quadros. O levantamento bibliográfico demonstrou que devido ao aumento de preditores como a presença de transtorno psiquiátrico associado, isolamento social, desemprego, uso de álcool, violência doméstica, estigma social, notícias negativas, doenças neurológicas, transtornos do sono, acesso restrito a serviços de saúde e outros, espera-se um aumento proporcional nas taxas de comportamento suicida durante e após o cenário atual.

Com base nisso, torna-se necessário que medidas de saúde sejam implementadas, com o objetivo de ajudar a manter a saúde psicológica e a boa qualidade de vida dos atingidos. Vale destacar que, a prática da quarentena e do isolamento social são essenciais para mitigar a disseminação da COVID-19, mas a preocupação com a saúde em geral não deve ser ignorada. Neste sentido, sugere-se que iniciativas com o objetivo de cuidar do sofrimento psíquico da população atingida sejam implementadas, além de elaborar estratégias voltadas às possíveis mudanças comportamentais de cada indivíduo respeitando as peculiaridades do contexto que está inserido.

São necessários mais estudos na área que contribuam para o reconhecimento precoce de fatores de risco que interferem diretamente no comportamento suicida. Diante do cenário atual, diversos preditores encontram-se exacerbados e isso reflete diretamente no aumento das taxas de suicídio esperadas a curto e longo prazo, criando assim uma possível pandemia "paralela" ocasionada pelo aumento dos índices de comportamento suicida.

Como sugestões para trabalhos futuros, faz-se necessário conduzir estudos para o aprofundamento dos impactos da COVID- 19 no comportamento suicida, ressaltando o reconhecimento e prevenção precoce dos fatores de risco, os quais se manifestam durante e após a pandemia. É importante que novos estudos foquem no desenvolvimento de estratégias de enfrentamento mais definidas diante dessa problemática, a fim de minimizar o impacto social, econômico e na saúde pública referente ao aumento do comportamento suicida.

\section{Referências}

Ammerman, B. A., Burke, T. A., Jacobucci, R., \& Mcclure, K. (2020). Preliminary investigation of the association between COVID-19 and suicidal thoughts and behaviors in the U.S. Journal Of Psychiatric Research, [2020], v. 134, p. 32-38, fev. 2021. Elsevier BV. http://dx.doi.org/10.1016/j.jpsychires.2020.12.037.

Aquila, I., Sacco, M. A., Ricci, C., Gratteri, S., Montebianco Abenavoli, L., Oliva, A., \& Ricci, P. (2020). The role of the COVID-19 pandemic as a risk factor for suicide: What is its impact on the public mental health state today? Psychological Trauma: Theory, Research, Practice and Policy, 12(S1), S120-S122. https://doi.org/10.1037/tra0000616.

Banerjee, D., Kosagisharaf, J. R., \& Sathyanarayana Rao, T. S. (2021). "The dual pandemic" of suicide and COVID-19: A biopsychosocial narrative of risks and prevention. Psychiatry Research, 295, 113577. https://doi.org/10.1016/j.psychres.2020.113577.

Bardin, L. (2011). Análise de conteúdo. Edições 70. 
Bastiampillai, T., Allison, S., Looi, J. C. L., Licinio, J., Wong, M.-L., \& Perry, S. W. (2020). The COVID-19 pandemic and epidemiologic insights from recession-related suicide mortality. Molecular Psychiatry, 25 (12), 3445-3447. https://doi.org/10.1038/s41380-020-00875-4.

Brietzke, E., Magee, T., Freire, R. C. R., Gomes, F. A., \& Milev, R. (2020). Three insights on psychoneuroimmunology of mood disorders to be taken from the COVID-19 pandemic. Brain, Behavior, \& Immunity - Health, 5, 100076. https://doi.org/10.1016/j.bbih.2020.100076.

Caballero-Domínguez, C. C., Jiménez-Villamizar, M. P., \& Campo-Arias, A. (2020). Suicide risk during the lockdown due to coronavirus disease (COVID19) in Colombia. Death Studies, 1-6. https://doi.org/10.1080/07481187.2020.1784312.

Campbell, A. M. (2020). An increasing risk of family violence during the Covid-19 pandemic: Strengthening community collaborations to save lives. Forensic Science International: Reports, 2, 100089. https://doi.org/10.1016/j.fsir.2020.100089.

Conejero, I., Nobile, B., Olié, E., \& Courtet, Ph. (2021). How Does COVID-19 Affect the Neurobiology of Suicide? Current Psychiatry Reports, 23(4). https://doi.org/10.1007/s11920-021-01227-x.

Cuellar, A., Mark, T. L., Sharfstein, S. S., \& Huskamp, H. A. (2020). How to Mitigate the Mental Health Care Consequences of the COVID-19 Financial Crisis. Psychiatric Services (Washington, D.C.), 71(12), 1317-1319. https://doi.org/10.1176/appi.ps.202000329.

Czeisler, M. É., Lane, R. I., Petrosky, E., Wiley, J. F., Christensen, A., Njai, R., Weaver, M. D., Robbins, R., Facer-Childs, E. R., Barger, L. K., Czeisler, C. A., Howard, M. E., \& Rajaratnam, S. M. W. (2020). Mental Health, Substance Use, and Suicidal Ideation During the COVID-19 Pandemic-United States, June 24-30, 2020. Morbidity and Mortality Weekly Report, 69(32), 1049-1057. https://doi.org/10.15585/mmwr.mm6932a1.

Deady, M., Tan, L., Kugenthiran, N., Collins, D., Christensen, H., \& Harvey, S. B. (2020). Unemployment, suicide and COVID-19: Using the evidence to plan for prevention. The Medical Journal of Australia, 213(4), 153-154.e1. https://doi.org/10.5694/mja2.50715.

Fitzpatrick, K. M., Harris, C., \& Drawve, G. (2020). How bad is it? Suicidality in the middle of the COVID-19 pandemic. Suicide \& Life-Threatening Behavior, 50(6), 1241-1249. https://doi.org/10.1111/sltb.12655.

Fountoulakis, K. N., Apostolidou, M. K., Atsiova, M. B., Filippidou, A. K., Florou, A. K., Gousiou, D. S., Katsara, A. R., Mantzari, S. N., PadouvaMarkoulaki, M., Papatriantafyllou, E. I., Sacharidi, P. I., Tonia, A. I., Tsagalidou, E. G., Zymara, V. P., Prezerakos, P. E., Koupidis, S. A., Fountoulakis, N. K., \& Chrousos, G. P. (2021). Self-reported changes in anxiety, depression and suicidality during the COVID-19 lockdown in Greece. Journal of Affective Disorders, 279, 624-629. https://doi.org/10.1016/j.jad.2020.10.061

Garcia, L. P., Sanchez, Z. M., Garcia, L. P., \& Sanchez, Z. M. (2020). Consumo de álcool durante a pandemia da COVID-19: Uma reflexão necessária para o enfrentamento da situação. Cadernos de Saúde Pública, 36(10). https://doi.org/10.1590/0102-311x00124520.

Gomes, I. S., \& Caminha, I. D. O. (2013). Guia para estudos de revisão sistemática: Uma opção metodológica para as ciências do movimento humano. Movimento (ESEFID/UFRGS), 20(1), 395. https://doi.org/10.22456/1982-8918.41542.

Gunnell, D., Appleby, L., Arensman, E., Hawton, K., John, A., Kapur, N., Khan, M., O'Connor, R. C., \& Pirkis, J. (2020). Suicide risk and prevention during the COVID-19 pandemic. The Lancet. Psychiatry, 7(6), 468-471. https://doi.org/10.1016/S2215-0366(20)30171-1.

Han, R. H., Schmidt, M. N., Waits, W. M., Bell, A. K. C., \& Miller, T. L. (2020). Planning for Mental Health Needs During COVID-19. Current Psychiatry Reports, 22(12). https://doi.org/10.1007/s11920-020-01189-6.

Hernández-Calle, D., Martínez-Alés, G., Mediavilla, R., Aguirre, P., Rodríguez-Vega, B., \& Bravo-Ortiz, M. F. (2020). Trends in Psychiatric Emergency Department Visits Due to Suicidal Ideation and Suicide Attempts During the COVID-19 Pandemic in Madrid, Spain. The Journal of Clinical Psychiatry, 81(5). https://doi.org/10.4088/JCP.20113419.

Jefsen, O. H., Rohde, C., Nørremark, B., \& Østergaard, S. D. (2020). COVID-19-related self-harm and suicidality among individuals with mental disorders Acta Psychiatrica Scandinavica, 142(2), 152-153. https://doi.org/10.1111/acps.13214.

Kawohl, W., \& Nordt, C. (2020). COVID-19, unemployment, and suicide. The Lancet. Psychiatry, 7(5), 389-390. https://doi.org/10.1016/S2215$0366(20) 30141-3$

Leaune, E., Samuel, M., Oh, H., Poulet, E., \& Brunelin, J. (2020). Suicidal behaviors and ideation during emerging viral disease outbreaks before the COVID19 pandemic: A systematic rapid review. Preventive Medicine, 141, 106264. https://doi.org/10.1016/j.ypmed.2020.106264.

Lennon, J. C. (2020). What lies ahead: Elevated concerns for the ongoing suicide pandemic. Psychological Trauma: Theory, Research, Practice and Policy, 12(S1), S118-S119. https://doi.org/10.1037/tra0000741.

Levi-Belz, Y., \& Aisenberg, D. (2020). Together we stand: Suicide risk and suicide prevention among Israeli older adults during and after the COVID-19 world crisis. Psychological Trauma: Theory, Research, Practice and Policy, 12(S1), S123-S125. https://doi.org/10.1037/tra0000667.

Mari, J. de J., \& Oquendo, M. A. (2020). Mental health consequences of COVID-19: The next global pandemic. Trends in Psychiatry and Psychotherapy, 42(3), 219-221. https://doi.org/10.1590/2237-6089-2020-0081.

McIntyre, R. S., \& Lee, Y. (2020). Projected increases in suicide in Canada as a consequence of COVID-19. Psychiatry Research, 290,113104 https://doi.org/10.1016/j.psychres.2020.113104.

Moutier, C. (2020). Suicide Prevention in the COVID-19 Era: Transforming Threat Into Opportunity. JAMA Psychiatry. https://doi.org/10.1001/jamapsychiatry.2020.3746.

Que, J., Yuan, K., Gong, Y., Meng, S., Bao, Y., \& Lu, L. (2020). Raising awareness of suicide prevention during the COVID-19 pandemic. Neuropsychopharmacology Reports, 40(4), 392-395. https://doi.org/10.1002/npr2.12141. 
Research, Society and Development, v. 10, n. 5, e59410515923, 2021

(CC BY 4.0) | ISSN 2525-3409 | DOI: http://dx.doi.org/10.33448/rsd-v10i5.15923

Rana, U. (2020). Elderly suicides in India: An emerging concern during COVID-19 pandemic. International Psychogeriatrics, 1-2. https://doi.org/10.1017/S1041610220001052.

Sheffler, J. L., Joiner, T. E., \& Sachs-Ericsson, N. J. (2021). The Interpersonal and Psychological Impacts of COVID-19 on Risk for Late-Life Suicide. The Gerontologist, 61(1), 23-29. https://doi.org/10.1093/geront/gnaa103.

Sher, L. (2020a). Are COVID-19 survivors at increased risk for suicide? Acta Neuropsychiatrica, 32(5), 270. https://doi.org/10.1017/neu.2020.21.

Sher, L. (2020b). Suicide research and prevention during and after the COVID-19 pandemic. Acta Psychiatrica Scandinavica, 142(5), 353-354. https://doi.org/10.1111/acps.13248.

Sher, L. (2020c). Psychiatric disorders and suicide in the COVID-19 era. QJM: An International Journal of Medicine, 113(8), 527-528. https://doi.org/10.1093/qjmed/hcaa204.

Stockwell, T., Andreasson, S., Cherpitel, C., Chikritzhs, T., Dangardt, F., Holder, H., Naimi, T., \& Sherk, A. (2021). The burden of alcohol on health care during COVID-19. Drug and Alcohol Review, 40(1), 3-7. https://doi.org/10.1111/dar.13143.

Thakur, V., \& Jain, A. (2020). COVID 2019-suicides: A global psychological pandemic. Brain, Behavior, and Immunity, 88, 952-953. https://doi.org/10.1016/j.bbi.2020.04.062.

Ventriglio, A., Watson, C., \& Bhugra, D. (2020). Pandemics, panic and prevention: Stages in the life of COVID-19 pandemic. The International Journal of Social Psychiatry, 66(8), 733-734. https://doi.org/10.1177/0020764020924449.

Wand, A. P. F., Zhong, B.-L., Chiu, H. F. K., Draper, B., \& De Leo, D. (2020). COVID-19: The implications for suicide in older adults. International Psychogeriatrics, 32(10), 1225-1230. https://doi.org/10.1017/S1041610220000770.

Wasserman, D., van der Gaag, R., \& Wise, J. (2020). The term "physical distancing" is recommended rather than "social distancing" during the COVID-19 pandemic for reducing feelings of rejection among people with mental health problems. European Psychiatry: The Journal of the Association of European Psychiatrists, 63(1), e52. https://doi.org/10.1192/j.eurpsy.2020.60.

Zalsman, G., Stanley, B., Szanto, K., Clarke, D. E., Carli, V., \& Mehlum, L. (2020). Suicide in the Time of COVID-19: Review and Recommendations. Archives of Suicide Research: Official Journal of the International Academy for Suicide Research, 24(4), 477-482. https://doi.org/10.1080/13811118.2020.1830242. 